\title{
Stability and feasibility of state constrained MPC without stabilizing terminal constraints
}

\author{
Andrea Boccia, Lars Grüne, Karl Worthmann \\ Control and Power Group, Electrical and Electronic Engineering, Imperial College \\ London. \\ Mathematical Institute, University of Bayreuth, 95440 Bayreuth, Germany. \\ Institut für Mathematik, Technische Universität Ilmenau, 98693 Ilmenau, Germany.
}

\begin{abstract}
In this paper we investigate stability and recursive feasibility of a nonlinear receding horizon control scheme without terminal constraints and costs but imposing state and control constraints. Under a local controllability assumption we show that every level set of the infinite horizon optimal value function is contained in the basin of attraction of the asymptotically stable equilibrium for sufficiently large optimization horizon $N$.

For stabilizable linear systems we show the same for any compact subset of the interior of the viability kernel. Moreover, estimates for the necessary horizon length $N$ are given via an analysis of the optimal value function at the boundary of the viability kernel.
\end{abstract}

Keywords: predictive control, optimal control, nonlinear control, linear systems, stability, state constraints, feasibility, optimal value functions

\section{Introduction}

Model predictive control (MPC) is a controller design technique relying on the iterative solution of optimal control problems. In this paper we study stability and recursive feasibility of nonlinear MPC schemes without stabilizing terminal constraints or costs. For such schemes, it is known that stability

Email address: a.boccia@imperial.ac.uk, lars.gruene@uni-bayreuth.de, karl.worthmann@tu-ilmenau.de (Andrea Boccia, Lars Grüne, Karl Worthmann) 
for sufficiently large optimization horizons can be deduced from controllability assumptions or - alternatively and almost equivalently — bounds on the optimal value functions, see Jadbabaie and Hauser (2005); Grimm et al. (2005); Tuna et al. (2006); Grüne (2009); Grüne et al. (2010); Grüne and Pannek (2011); Worthmann (2011).

The present paper extends this body of literature by taking into account state constraints without assuming viability of the state constraint set or boundedness of the optimal value function on this set or its viability kernel. Avoiding the viability assumption is important since computing viable state constraint sets is typically difficult if not impossible for complex systems. Our analysis thus ensures proper functioning of MPC also in this case. Allowing for unbounded optimal value functions is important in order to study the behaviour of MPC schemes in a neighborhood of the boundary of the stabilizable set, from which it may take arbitrarily long to control the system to the desired equilibrium and thus the optimal value function can have arbitrarily large values.

In the first part of the paper we consider general nonlinear systems and assume a local controllability assumption in a neighbourhood of the equilibrium to be stabilized. Under this condition, we first analyse the behaviour of the closed loop on level sets $V_{\infty}^{-1}[0, C]$ of the infinite horizon optimal value function. Using a technique similar to Primbs and Nevistić (2000) we obtain recursive feasibility and an adaptation of an argument from Grimm et al. (2005) yields asymptotic stability with $V_{\infty}^{-1}[0, C]$ contained in the basin of attraction, provided the optimization horizon $N$ is suffiently large. Moreover, quantitative estimates on the necessary length of $N$ are given. This result is then extended to compact sets lying in the domain of $V_{\infty}$ and avoiding suitable defined exceptional regions $\mathcal{O}$. Overall, this part of the paper can be seen as a (discrete time) extension of Jadbabaie and Hauser (2005) to the state constrained case and with additional quantitative estimates for $N$.

In the second part of the paper we specialize the results to the linear quadratic case with convex constraints. We show that in this setting any compact subset $K$ in the interior of the viability kernel is contained in the basin of attraction for sufficiently large $N$ and give an estimate of $N$ in terms of the distance of $K$ to the boundary of the viability kernel. These quantitative results rely on an estimate of the growth of the optimal value function $V_{\infty}$ at the boundary of the viability kernel which we obtained adapting a technique from Gondhalekar et al. (2009). A particularly nice case appears when $V_{\infty}$ is bounded on the viability kernel and we show that this prop- 
erty implies stationarity of the feasible sets in the sense of (Kerrigan, 2000, Chapter 5).

The paper is organized as follows. After describing the setting in Section 2 , Section 3 contains the nonlinear asymptotic stability and feasibility results. The specialization to linear systems is presented in Section 4 and conclusions are given in Section 5.

Notation: $\mathbb{R}$ and $\mathbb{N}$ denote real and natural numbers, respectively. $\mathbb{N}_{0}:=$ $\mathbb{N} \cup\{0\}$ and $\mathbb{R}_{\geq 0}$ indicates non-negative real numbers. The Euclidean norm in $\mathbb{R}^{n}$ is written as $|\cdot|$ while given a matrix $M \in \mathbb{R}^{n \times m},\|M\|:=\sup _{|x| \leq 1}|M x|$. $\mathbb{B}$ denotes the closed unit ball in $\mathbb{R}^{n}$. Given a set $S \subset \mathbb{R}^{n}, \bar{S}$ denotes its closure, int $S$ its interior and $\partial S:=\bar{S} \backslash$ int $S$ its boundary. Furthermore, a continuous function $\eta: \mathbb{R}_{\geq 0} \rightarrow \mathbb{R}_{\geq 0}$ is said to be of class $\mathcal{K}$ if it is strictly increasing and satisfies $\eta(0)=0$. If $\eta \in \mathcal{K}$ is also unbounded, $\eta$ is called a class $\mathcal{K}_{\infty}$-function. A function $\beta: \mathbb{R}_{\geq 0} \times \mathbb{R}_{\geq 0} \rightarrow \mathbb{R}_{\geq 0}$ is called $\mathcal{K} \mathcal{L}$-function if it is continuous, satisfies $\beta(\cdot, t) \in \mathcal{K}_{\infty}, t \in \mathbb{R}_{\geq 0}$, is strictly decreasing in its second argument for all $r>0$, and $\lim _{t \rightarrow \infty} \beta(r, t)=0$ holds.

\section{Model Predictive Control}

We consider discrete time systems governed by the system dynamics

$$
x^{+}=f(x, u)
$$

where $f: \mathbb{R}^{n} \times \mathbb{R}^{m} \rightarrow \mathbb{R}^{n}$ is a map which determines the successor state $x^{+}$in dependence of the current state $x \in \mathbb{R}^{n}$ and the control input $u \in \mathbb{R}^{m}$. The state trajectory emanating from initial state $x_{0}$ and generated by the control sequence $u=(u(k))_{k \in \mathbb{N}_{0}}$ is denoted by $x_{u}\left(k ; x_{0}\right), k \in \mathbb{N}_{0}$. Here, the trajectory $x_{u}(\cdot)=x_{u}\left(\cdot ; x_{0}\right)$ is defined iteratively by $x_{u}\left(k+1 ; x_{0}\right)=f\left(x_{u}\left(k ; x_{0}\right), u(k)\right)$ and $x_{u}\left(0, x_{0}\right)=x_{0}$. Constraints for the state $x$ and the control $u$ are modeled by a suitably chosen subset $\mathcal{E} \subseteq \mathbb{R}^{n} \times \mathbb{R}^{m}$, i.e., we require

$$
(x, u) \in \mathcal{E} \text {. }
$$

Hence, for a given set $\mathcal{E}$, the set of admissible states is given by the projection of the set $\mathcal{E}$ on the state space $\mathbb{R}^{n}$, i.e.

$$
X:=\operatorname{proj}_{\mathbb{R}^{n}}(\mathcal{E})=\left\{x \in \mathbb{R}^{n}: \exists u \in \mathbb{R}^{m} \text { s.t. }(x, u) \in \mathcal{E}\right\} .
$$

Furthermore, for a given admissible state $x \in X$, the control constraints can be represented by $U(x):=\left\{u \in \mathbb{R}^{m}:(x, u) \in \mathcal{E}\right\}$. Using these definitions the concept of an admissible control sequence can be defined as follows. 
Definition 1 (Admissible control sequence). A sequence of control values $u=(u(0), u(1), \ldots, u(N-1))$ is called admissible for $x_{0} \in X$ and $N \in$ $\mathbb{N} \cup\{\infty\}$ if the conditions

$$
f\left(x_{u}\left(k ; x_{0}\right), u(k)\right) \in X \quad \text { and } \quad u(k) \in U\left(x_{u}\left(k ; x_{0}\right)\right)
$$

hold for all $k \in\{0,1, \ldots, N-1\}$. The set of all admissible control sequences of length $N \in \mathbb{N} \cup\{\infty\}$ is denoted by $\mathcal{U}^{N}\left(x_{0}\right)$.

Let $x^{\star} \in X$ be a (controlled) equilibrium, i.e. there exists $u^{\star} \in U\left(x^{\star}\right)$ such that $f\left(x^{\star}, u^{\star}\right)=x^{\star}$ holds. Our goal is to find a static state feedback $\mu: \mathbb{R}^{n} \rightarrow$ $\mathbb{R}^{m}$ and a basin of attraction $\mathcal{S} \subseteq X$ such that the resulting closed loop $x^{+}=$ $f(x, \mu(x))$ is asymptotically stable w.r.t. the desired equilibrium $x^{\star}$. This means that for any initial state $x_{0} \in \mathcal{S}$ the closed loop trajectory $x_{\mu}\left(k ; x_{0}\right)$, $k \in \mathbb{N}_{0}$, generated by

$$
x_{\mu}\left(k+1 ; x_{0}\right)=f\left(x_{\mu}\left(k ; x_{0}\right), \mu\left(x_{\mu}\left(k ; x_{0}\right)\right)\right), \quad x_{\mu}\left(0 ; x_{0}\right)=x_{0},
$$

remains feasible, i.e., $\left(x_{\mu}\left(k ; x_{0}\right), \mu\left(x_{\mu}\left(k ; x_{0}\right)\right)\right) \in \mathcal{E}$ holds for all $k \in \mathbb{N}_{0}$, and satisfies the estimate $\left|x_{\mu}\left(k ; x_{0}\right)-x^{\star}\right| \leq \beta\left(\left|x_{0}-x^{\star}\right|, k\right), k \in \mathbb{N}_{0}$, for some $\mathcal{K} \mathcal{L}$-function $\beta$.

In MPC, the feedback values $\mu(x)$ are computed by solving optimal control problems. To this end, running costs $\ell: \mathbb{R}^{n} \times \mathbb{R}^{m} \rightarrow \mathbb{R}_{\geq 0}$ satisfying $\ell\left(x^{\star}, u^{\star}\right)=0$ and

$$
\underline{\eta}\left(\left|x-x^{\star}\right|\right) \leq \ell^{\star}(x):=\inf _{u \in \mathcal{U}^{1}(x)} \ell(x, u) \leq \bar{\eta}\left(\left|x-x^{\star}\right|\right) \quad \forall x \in X
$$

for two $\mathcal{K}_{\infty}$-functions $\eta, \bar{\eta}$ are defined. The corresponding cost function $J_{N}: \mathbb{R}^{n} \times\left(\mathbb{R}^{m}\right)^{N} \rightarrow \mathbb{R}_{\geq 0}$ and optimal value function $V_{N}: \mathbb{R}^{n} \rightarrow \mathbb{R}_{\geq 0} \cup\{+\infty\}$ are given by

$$
J_{N}(x, u):=\sum_{k=0}^{N-1} \ell\left(x_{u}(k ; x), u(k)\right) \quad \text { and } \quad V_{N}(x):=\inf _{u \in \mathcal{U}^{N}(x)} J(x, u)
$$

for $N \in \mathbb{N} \cup\{\infty\}, x \in X$ and $u \in \mathcal{U}^{N}(x)$ with the convention $V_{N}(x)=+\infty$ if $x \notin X$ or $\mathcal{U}^{N}(x)=\emptyset$. In principle, the stabilization problem could be solved by solving the optimal control problem for $N=\infty$. However, solving optimal control problems on an infinite time horizon is, in general, computationally hard. This explains why we pursue a different approach: model predictive control (MPC), also termed receding horizon control. Fixing a finite prediction horizon (or optimization horizon) $N$ and setting $x_{\mu}\left(0 ; x_{0}\right):=x_{0}, k:=0$, the MPC loop is as follows: 
1. Measure $x=x_{\mu}\left(k ; x_{0}\right)$, solve the optimal control problem

$$
\min _{u \in \mathcal{U}^{N}(x)} J_{N}(x, u)
$$

and denote a respective minimizing control sequence ${ }^{1}$ by $u^{\star} \in \mathcal{U}^{N}(x)$.

2. Define the MPC feedback value by $\mu_{N}(x):=u^{\star}(0)$.

3. Apply the control $\mu_{N}(x)$ to the system, set $k:=k+1$ and go to 1 .

Remark 2. In this paper we consider nominal $M P C$, i.e., we assume that $x_{\mu}\left(k ; x_{0}\right)$ resulting from the MPC algorithm satisfies (4) with $\mu=\mu_{N}$. We remark that additional conditions would be needed in order to rigorously analyse the effect of perturbations, as, e.g., discussed in Sections 8.8 and 8.9 of Grüne and Pannek (2011). For the sake of brevity we refrain from addressing this issue in this paper.

The MPC iteration yields a closed loop trajectory for the implicitly defined MPC feedback law $\mu_{N}: X \rightarrow \mathbb{R}^{m}$. However, while for $N=\infty$ stability of the nominal closed loop follows by standard Lyapunov arguments, due to the truncation of the optimization horizon stability, feasibility, and optimality may get lost for finite $N$, see, e.g., Raff et al. (2006).

\section{Recursive Feasibility and Asymptotic Stability}

In order to guarantee that the optimal control problem in Step 1 of the MPC loop is feasible, we need to ensure $\mathcal{U}^{N}(x) \neq \emptyset$ for $x=x_{\mu_{N}}\left(k ; x_{0}\right)$, $k \in \mathbb{N}_{0}$. This problem can be solved by incorporating suitable terminal constraints and costs in the optimal control problem to be solved in each MPC step. However, the construction of such stabilizing constraints may be challenging and their use may considerably reduce the operating range of the MPC scheme, cf. (Grüne and Pannek, 2011, Chapter 8) or Mayne (2013) for a detailed discussion. Hence, we want to analyse the scheme without additional terminal constraints or costs. In particular, feasibility of the MPC algorithm in each step and asymptotic stability of the resulting closed loop has to be ensured. To this end, the following local controllability condition is employed.

\footnotetext{
${ }^{1}$ Whenever $\mathcal{U}^{N}(x) \neq \emptyset$, existence of a minimizer $u^{\star} \in \mathcal{U}^{N}(x)$ satisfying $J_{N}\left(x, u^{\star}\right)=$ $V_{N}(x)$ is assumed in order to avoid technical difficulties.
} 
Assumption 1. There exists a neighbourhood $\mathcal{N}$ of $x^{\star}$ and a positive constant $\gamma \in \mathbb{R}$ such that

$$
V_{\infty}(x) \leq \gamma \cdot \ell^{\star}(x), \quad \forall x \in \mathcal{N} \cap X .
$$

Remark 3. (i) The name controllability condition stems from the fact that the inequality $V_{\infty}(x)<C$ requires the system to be controllable to $x^{\star}$ sufficienty fast, since otherwise (5) implies $V_{\infty}(x)=+\infty$. For the particular form of the bound $\gamma$ assumed above, for instance the exponential controllability assumption w.r.t. $\ell($.$) used in (Grüne and Pannek, 2011, Chapter 6)$ would be sufficient. However, note that in contrast to Grüne and Pannek (2011) here we only require the inequality to hold locally around $x^{\star}$.

(ii) The condition on $V_{\infty}$ implies the analogous inequalities for $V_{N}, N \in$ $\mathbb{N}$. These inequalities could be replaced by inequalities in which $\gamma$ depends on $N$, thus allowing for less conservative estimates, cf. Worthmann (2011). However, in order to keep the presentation simple, in this paper we will work with the assumption on $V_{\infty}$ and a single $\gamma$.

In order to formalize recursive feasibility, some notation is needed. The feasible set for a horizon length $N \in \mathbb{N} \cup\{\infty\}$ is defined as

$$
\mathcal{F}_{N}:=\left\{x \in X: \mathcal{U}^{N}(x) \neq \emptyset\right\}
$$

The set $\mathcal{F}_{\infty}$ is also called viability kernel. Note that the definition immediately implies $\mathcal{F}_{N_{2}} \subseteq \mathcal{F}_{N_{1}}$ for all $N_{1}, N_{2} \in \mathbb{N}$ with $N_{1} \leq N_{2}$ and $\mathcal{F}_{\infty} \subseteq \mathcal{F}_{N}$ for all $N \in \mathbb{N}$.

A set $\mathcal{C} \subseteq X$ is said to be (controlled) forward invariant or viable if, for each $x \in \mathcal{C}$, there exists $u \in U(x)$ such that $f(x, u) \in \mathcal{C}$ holds. Observe that every forward invariant set $\mathcal{C} \subseteq X$ satisfies the inclusion $\mathcal{C} \subseteq \mathcal{F}_{\infty}$ and that the set of admissible states $X$ is, in general, much larger than the viability kernel $\mathcal{F}_{\infty}$. Methods which can be used in order to compute invariant sets can be found in Blanchini and Miani (2008). The set $\mathcal{C}$ is said to be recursively feasible if it is forward invariant with respect to the feedback law $\mu_{N}$, that is $\mu_{N}(x) \in U(x)$ and $f\left(x, \mu_{N}(x)\right) \in \mathcal{C}$ for all $x \in \mathcal{C}$.

\subsection{Asymptotic Stability on Level Sets}

Ideally we would like the basin of attraction $\mathcal{S}$ to coincide with $\mathcal{F}_{\infty}$ since the viability kernel is the maximal set on which an admissible feedback can be defined. However, this is, in general, not possible. The reason for this is 
that the closer we get to the boundary of $\mathcal{F}_{\infty}$, the more costly (in the sense of our objective $J_{N}$ ) it may become to steer the system to $x^{\star}$ and if this happens then the optimization criterion may lead the MPC closed loop to stay near the boundary of $\mathcal{F}_{\infty}$ instead of approaching $x^{\star}$. Hence, a central task considered in this paper is to estimate the basin of attraction $\mathcal{S} \subseteq \mathcal{F}_{\infty}$ and - conversely - given a set $K \subseteq \mathcal{F}_{\infty}$ to estimate an optimization horizon $N$ such that $K \subseteq \mathcal{S}$ is guaranteed. We point out that in general the domain of $V_{\infty}, \operatorname{dom} V_{\infty}:=\left\{x: V_{\infty}(x)<+\infty\right\}$, is strictly contained in $\mathcal{F}_{\infty}$, cf. the example after Proposition 12.

As a first step, we consider the problem of determining a recursively feasible set. To this end, for a given horizon length $N \in \mathbb{N} \cup\{\infty\}$ and a positive constant $C$ define the level set

$$
V_{N}^{-1}[0, C]:=\left\{x \in X: V_{N}(x) \leq C\right\} .
$$

Since the running costs are supposed to satisfy (5), existence of the lower bound

$$
M:=\inf _{x \in X \backslash \mathcal{N}} \ell^{\star}(x)>0
$$

is ensured. Then, for every $x \in V_{N}^{-1}[0, C] \backslash \mathcal{N}$, the inequality

$$
V_{N}(x) \leq \frac{C}{M} \cdot M \leq \frac{C}{M} \cdot \ell^{\star}(x)
$$

holds. The parameter $C$ can be chosen sufficiently large such that the inequality

$$
V_{N}(x) \leq \gamma \cdot \ell^{\star}(x) \leq \gamma \cdot \sup _{x \in \mathcal{N} \cap X} \ell^{\star}(x) \leq C
$$

holds for all $x \in \mathcal{N} \cap X$. Summarizing, a constant $\beta=\beta(C, M, \gamma)$ only depending on Assumption 1, Condition (5), and a parameter $C$ can be found satisfying

$$
V_{N}(x) \leq \beta \cdot \ell^{\star}(x) \quad \forall x \in V_{N}^{-1}[0, C] \quad \text { and } \quad \mathcal{N} \cap X \subseteq V_{N}^{-1}[0, C] .
$$

This in particular shows that Assumption 1 can be extended to arbitrary level sets $V_{N}^{-1}[0, C]$. This fact is exploited in order to prove Theorem 4.

Theorem 4. Let Assumption 1 and Inequality (5) be satisfied. Take any positive real number $C$ satisfying (8) and let $M$ be defined as in (7). In addition, choose $N_{0} \in \mathbb{N}$ such that the inequalities

$$
C\left(\frac{\beta-1}{\beta}\right)^{N_{0}-1}<M \quad \text { and } \quad 1-\alpha_{N_{0}}>0
$$


are satisfied with $\beta:=\max \{C / M, \gamma, 2\}$ and $\alpha_{N}:=\beta^{2}\left(\frac{\beta-1}{\beta}\right)^{N}$. Then, for every $N \geq N_{0}$ and every $x \in V_{N}^{-1}[0, C]$, we have

$$
V_{N}\left(f\left(x, \mu_{N}(x)\right)\right) \leq V_{N}(x)-\left(1-\alpha_{N}\right) \ell^{\star}(x) .
$$

In particular $V_{N}(\cdot)$ is a Lyapunov function on the recursively feasible set $V_{N}^{-1}[0, C]$ which implies recursive feasibility and asymptotic stability of the MPC closed loop.

Proof. The proof is an adaptation of the arguments developed in Grüne (2012) to our setting. In particular, Variant II from Section 3.2 of this paper is used, whose idea was taken from Tuna et al. (2006). Take any $x \in V_{N}^{-1}[0, C]$. Then $V_{N}(x) \leq C$ and by hypothesis there exists an admissible control sequence $u^{\star} \in \mathcal{U}^{N}(x)$ such that $V_{N}(x)=J_{N}\left(x, u^{\star}\right)$. If we define

$$
\ell_{k}:=\ell\left(x_{u^{\star}}(k ; x), u^{\star}(k)\right) \quad \text { for } \quad k \in\{0,1, \ldots, N-1\},
$$

then $V_{N}(x)$ can be written as $V_{N}(x)=\sum_{k=0}^{p-1} \ell_{k}+V_{N-p}\left(x_{u^{\star}}(p ; x)\right)$ for any $p=$ $0,1, \ldots, N-1$. This implies $V_{N-p}\left(x_{u^{\star}}(p ; x)\right) \leq C$, i.e., $x_{u^{\star}}(p ; x) \in V_{N-p}^{-1}[0, C]$. Since $\beta$ only depends on $C, \gamma$, and $M$ (and not on the optimization horizon) from (9) we obtain the inequality $V_{N-p}\left(x_{u^{\star}}(p ; x)\right) \leq \beta \ell_{p}$. Therefore

$$
V_{N}(x)=\sum_{k=0}^{N-1} \ell_{k} \leq \sum_{k=0}^{p-1} \ell_{k}+\beta \ell_{p}
$$

If $f\left(x, \mu_{N}(x)\right)$ is feasible, i.e., if $f\left(x, \mu_{N}(x)\right) \in \mathcal{F}_{N}$ holds or, equivalently, $\mathcal{U}^{N}\left(f\left(x, \mu_{N}(x)\right)\right) \neq \emptyset$, we obtain the inequality

$$
\begin{aligned}
V_{N}\left(f\left(x, \mu_{N}(x)\right)\right) & \leq \sum_{k=1}^{N-2} \ell_{k}+V_{2}\left(x_{u^{\star}}(N-1 ; x)\right) \\
& =V_{N}(x)-\ell_{0}-\ell_{N-1}+V_{2}\left(x_{u^{\star}}(N-1 ; x)\right) .
\end{aligned}
$$

In general, however, without additional hypotheses, we cannot guarantee feasibility of $f\left(x, \mu_{N}(x)\right)$. Still, by setting $V_{2}\left(x_{u_{\star}}(N-1 ; x)\right)=+\infty$ in case of infeasibility we can extend (13) to this case.

We keep this in mind and show $x_{u^{\star}}(N-1 ; x) \in \mathcal{N} \cap X$ and, thus, $V_{2}\left(x_{u^{\star}}(N-1 ; x)\right)<\infty$. Indeed, by $(12), \sum_{k=p+1}^{N-1} \ell_{k} \leq(\beta-1) \ell_{p}$ holds which implies

$$
\sum_{k=p}^{N-1} \ell_{k} \geq\left(\frac{\beta}{\beta-1}\right) \sum_{k=p+1}^{N-1} \ell_{k} \geq\left(\frac{\beta}{\beta-1}\right)^{2} \sum_{k=p+2}^{N-1} \ell_{k} \geq \ldots \geq\left(\frac{\beta}{\beta-1}\right)^{N-p-1} \ell_{N-1}
$$


for $p \in\{0,1, \ldots, N-1\}$. When $p=0$, since $x \in V_{N}^{-1}[0, C]$, we obtain

$$
\begin{aligned}
C & \geq V_{N}(x) \geq\left(\frac{\beta}{\beta-1}\right)^{N-1} \ell_{N-1}, \\
\beta \cdot \ell^{\star}(x) & \geq V_{N}(x) \geq\left(\frac{\beta}{\beta-1}\right)^{N-1} \ell_{N-1} .
\end{aligned}
$$

According to our choice of $N$ Inequality (14) implies $\ell^{\star}\left(x_{u^{\star}}(N-1 ; x)\right) \leq$ $\ell_{N-1}<M$ and, in view of $x_{u_{\star}}(N-1 ; x) \in X$ and $(7), x_{u^{\star}}(N-1 ; x) \in \mathcal{N} \cap \bar{X}$ where our local Assumption 1 can be invoked. Consequently feasibility and $V_{2}\left(x_{u^{\star}}(N-1 ; x)\right) \leq \gamma \ell_{N-1} \leq \beta \ell_{N-1}$ hold. A further appeal to (13) and (15) now gives

$$
V_{N}\left(f\left(x, \mu_{N}(x)\right)\right) \leq V_{N}(x)-\ell_{0}+(\beta-1) \ell_{N-1} \leq V_{N}(x)-\left(1-\alpha_{N}\right) \ell^{\star}(x),
$$

i.e. Inequality (11) and recursive feasibility of the level set $V_{N}^{-1}[0, C]$. From this and the bounds on $V_{N}$ induced by (5) and (9), the Lyapunov function property of $V_{N}$ and asymptotic stability follow by standard arguments, see, e.g. (Grüne, 2009, Section 5).

Remark 5. The optimization horizon $N_{0}$ guaranteeing stability in Theorem 4 grows like $2(C / M) \ln C$ as $C \rightarrow+\infty$. Indeed the horizon $N$ must satisfy

$$
N>\frac{2 \ln \beta}{\ln \beta-\ln (\beta-1)}
$$

and $\beta \sim(C / M)$ as $C \rightarrow \infty .^{2}$ A more careful analysis shows that this bound can be improved by a factor 2: let $\widetilde{\mathcal{N}}$ be a neighbourhood of the origin such that $\widetilde{\mathcal{N}} \cap X$ is controlled forward invariant. ${ }^{3}$ Define $\widetilde{M}:=\inf _{x \in X \backslash \widetilde{\mathcal{N}}} \ell^{\star}(x)$ and assume that the horizon $N$ only satisfies the first inequality in (10), now with respect to the constant $\widetilde{M}$. Then feasibility of the MPC closed loop trajectory is ensured - as proved in Theorem 4- since $x_{u_{\star}}(N-1 ; x) \in \tilde{\mathcal{N}} \cap X$ which is forward invariant. Since feasibility is now ensured, the estimates from (Grüne

\footnotetext{
${ }^{2}$ We use the notation $f(x) \sim g(x)$ as $x \rightarrow \infty$ to indicate that the functions $f(\cdot)$ and $g(\cdot)$ have asymptotically the same behaviour, i.e., that $\lim _{x \rightarrow \infty} \frac{f(x)}{g(x)}=1$ holds.

${ }^{3}$ Such a neighborhood $\widetilde{\mathcal{N}}$ exists under the assumptions of Theorem 4 as one may define $\widetilde{\mathcal{N}}$ as the interior of a sublevel set of $V_{N}$.
} 
et al., 2010, Section 6) can be applied to get the improved value

$$
\alpha_{N}=\frac{(\beta-1)^{N}}{\beta^{N-1}-(\beta-1)^{N-1}}
$$

which is smaller than 1 (as required) when $N>2+\frac{\ln (\beta-1)}{\ln \beta-\ln (\beta-1)}$. This bound for the optimization horizon $N$ now behaves asymptotically as $(C / M) \ln C$ for $C \rightarrow+\infty$.

Often more restrictive controllability conditions are assumed in order to ensure asymptotic stability, see, e.g. Tuna et al. (2006) or Grüne (2012) where our local Assumption 1 was assumed on a (controlled) invariant subset of the viability kernel. We like to point out that no 'viability' conditions - such as forward invariance - nor regularity hypotheses on the dynamics $f(\cdot, \cdot)$ and the control constraint set $U(\cdot)$ are imposed on $X$ in this section.

\subsection{Global stability}

Theorem 4 implies that for each compact set $K \subseteq X$ satisfying $C:=$ $\sup V_{\infty}(K)<\infty$ the MPC controller yields asymptotic stability for $N \geq N_{0}$ with a basin of attraction $\mathcal{S} \supseteq V_{N}^{-1}[0, C] \supseteq K$. In order to analyse which kind of sets $K$ have this property, we consider the set $V_{\infty}^{-1}[0,+\infty)=\{x \in$ $\left.X: V_{\infty}(x)<+\infty\right\}$ and the decreasing family of sets $V_{\infty}^{-1}[n,+\infty) \supseteq V_{\infty}^{-1}[n+$ $1,+\infty)$ with varying $n \in \mathbb{N}$. For these sets we consider the set valued limit

$$
\mathcal{O}:=\lim _{n \rightarrow \infty} V_{\infty}^{-1}[n,+\infty)=\bigcap_{n \in \mathbb{N}} \overline{V_{\infty}^{-1}[n,+\infty)},
$$

cf. (Aubin and Frankowska, 1990, Section 1.1). In many cases of interest the set $\mathcal{O}$ has zero measure. If, for example, the value function is uniformly bounded or continuous on the set $V_{\infty}^{-1}[0,+\infty)$, then the set $V_{\infty}^{-1}[0,+\infty) \cap \mathcal{O}$ is empty. Later on, conditions ensuring one of these two stipulations are investigated for linear systems, see Section 4.

The relevance of the set $\mathcal{O}$ for MPC stems from the following claim: take any compact set $K \subset V_{\infty}^{-1}[0,+\infty) \backslash \mathcal{O}$. Then we claim that there exists $C \in \mathbb{R}_{>0}$ such that

$$
V_{\infty}^{-1}[0, C] \supseteq K
$$

Suppose to the contrary, it would exist a convergent sequence $\left(x_{n}\right)_{n \in \mathbb{N}_{0}} \subset K$ such that $x_{n} \rightarrow x \in K$ and $V_{\infty}\left(x_{n}\right)>n$. Thus $x_{n} \in V_{\infty}^{-1}[n,+\infty)$ and $x \in \mathcal{O}$. This is not possible since $x \in K$. 
The following theorem shows the consequences of this claim for the MPC controller. Its proof is an immediate consequence of the facts stated so far in this section.

Theorem 6. Let Assumption 1 and Condition (5) be satisfied and $K \subset$ $V_{\infty}^{-1}[0,+\infty) \backslash \mathcal{O}$ be a compact set. Then there exists $N_{K} \in \mathbb{N}$ such that for each $N \geq N_{K}$ the MPC closed loop is recursively feasible and asymptotically stable with basin of attraction $\mathcal{S} \supseteq K$.

Theorem 6 provides a nonlinear extension of the linear results shown in Primbs and Nevistić (2000). It tells us that, for a sufficiently large horizon, the MPC algorithm provides a recursively feasible and asymptotically stable closed loop on every compact set in which the value function is finite, as long as we avoid 'small' areas of bad behaviour (close to $\mathcal{O}$ ). Note that Theorem 6 is also applicable if state constraints are present and thus extends Jadbabaie and Hauser (2005). Compared to these references, the main additional ingredient is the quantitative information on the upper bound on $V_{\infty}$ provided locally by Assumption 1 and globally by the requirement $K \subset V_{\infty}^{-1}[0,+\infty) \backslash \mathcal{O}$.

The set $\mathcal{O}$ can comprise points $x$ satisfying $V_{\infty}(x)=+\infty$, but may also contain points $x$ which are controllable to $x^{\star}$ in finite time with $V_{\infty}(x)<\infty$. The latter situation is shown in the following example.

Example 7. Consider the one dimensional system $x^{+}=u x^{2}+(1-u)\left(x-\frac{3}{2}\right)$ with $X=\left[-\frac{1}{2}, 2\right], U=\{1\} \cup\{0\}$ and equilibrium $x^{\star}=0$. Here, for a cost function, say $\ell(x, u):=|x|$, the set $V_{\infty}^{-1}[0,+\infty)$ is equal to $X$. Nevertheless, the set $\mathcal{O}$ is nonempty since $\mathcal{O}=\{1\}$ holds and $V_{\infty}(1)$ is finite, cf. Figure 1.

\section{Linear Systems}

This section is dedicated to linear constrained systems

$$
x^{+}=A x+B u, \quad(x, u) \in \mathcal{E} .
$$

For this class of systems we will be able to provide more precise estimates for the constants involved in the general nonlinear results of the last section. Moreover, we will be able to characterize the "exceptional set" $\mathcal{O}$ in more detail and investigate the relation between the stabilizable set $\mathcal{S}$ and the viability kernel $\mathcal{F}_{\infty}$. 


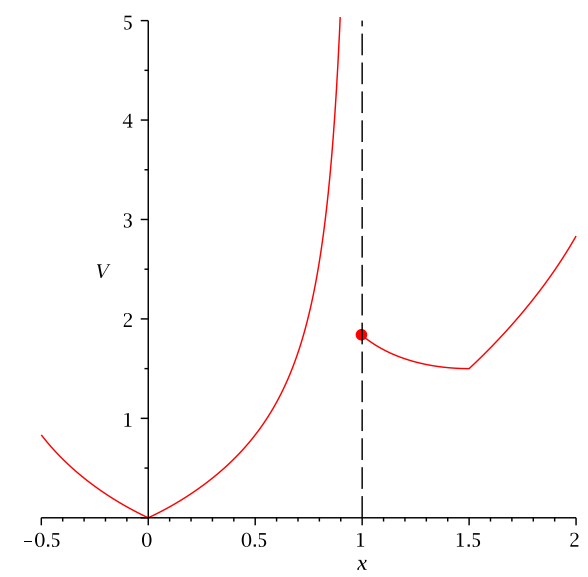

Figure 1: Illustration of the value function $V_{\infty}(\cdot)$ for Example 7.

Like for nonlinear systems, we will base our analysis on the controllability Assumption 1. It should be noted that for linear systems an alternative methodology for ensuring stability and recursive feasibility of the MPC closed loop is available, cf. Primbs and Nevistic (2000). However, this approach requires precise knowledge on the growth of the value function and can, thus, be seldomly applied if constraints are present. In contrast to that, techniques based on Assumption 1 can be applied since this condition is significantly easier to verify. Below, we prove that Assumption 1 can always be ensured for a large class of linear constrained systems. To this end, we make the folowing two assumptions.

Assumption 2. The constraint set $\mathcal{E}$ is convex, compact, and contains the origin $(0,0)$ in its interior.

Assumption 3. The linear system described by the pair $(A, B)$ is stabilizable.

\subsection{Characterization of the Viability Kernel for Linear Systems}

In the next two propositions we characterize the viability kernel $\mathcal{F}_{\infty}$ in order to gain insight into the structure of the set $\mathcal{S}$ on which the MPC feedback law $\mu_{N}$ stabilizes the system. While similar statements can be found in the viability literature, we decided to give sketches of the proofs in order to keep the paper self contained. 
Proposition 8. Consider the linear system (L) with constraint $\mathcal{E}$ that satisfies Assumption 2. The viability kernel $\mathcal{F}_{\infty}$, and all the feasible sets $\mathcal{F}_{N}$, $N \in \mathbb{N}$, are convex and compact sets.

Proof. Let $N \in \mathbb{N} \cup\{\infty\}$. In order to prove convexity, consider $x_{1}, x_{2} \in \mathcal{F}_{N}$. Then there are control sequences $u_{i}(\cdot) \in U^{N}\left(x_{i}\right)$ with $x_{u_{i}}\left(k ; x_{i}\right) \in \mathcal{F}_{N}$ for $i \in\{1,2\}$ and all $k=1,2, \ldots, N-1$. For any $\lambda \in[0,1]$, linearity of the dynamics implies

$$
x_{\lambda u_{1}+(1-\lambda) u_{2}}\left(k ; \lambda x_{1}+(1-\lambda) x_{2}\right)=\lambda x_{u_{1}}\left(k ; x_{1}\right)+(1-\lambda) x_{u_{2}}\left(k ; x_{2}\right)
$$

for all $k=0,1, \ldots, N-1$. Then convexity of $\mathcal{E}$ implies $\left(x_{\lambda u_{1}+(1-\lambda) u_{2}}\left(k ; \lambda x_{1}+\right.\right.$ $\left.\left.(1-\lambda) x_{2}\right), \lambda u_{1}(k)+(1-\lambda) u_{2}(k)\right) \in \mathcal{E}$ and thus $\lambda x_{1}+(1-\lambda) x_{2} \in \mathcal{F}_{N}$.

Compactness follows from the fact that the sets $\mathcal{F}_{N}$ are closed because $\mathcal{E}$ is closed and the dynamics is continuous, and bounded because $\mathcal{F}_{N} \subseteq X=$ $\operatorname{proj}_{\mathbb{R}^{n}}(\mathcal{E})$ and $X$ is bounded.

Proposition 9. Consider the viability kernel $\mathcal{F}_{\infty}$ given by (6) and linear dynamic as in (L). Let Assumption 2 be satisfied. Then, the following assertions hold.

(a) The set $\lambda \mathcal{F}_{\infty}$ is forward invariant for any $\lambda \in[0,1]$. More precisely take any $\lambda \in[0,1]$ and $x \in \lambda \mathcal{F}_{\infty}$, there exists an admissible control sequence $u=(u(k))_{k \in \mathbb{N}} \in \mathcal{U}^{\infty}(x)$ such that

$$
\left(x_{u}(k ; x), u(k)\right) \in \lambda \mathcal{E} \subseteq \mathcal{E} \quad \text { and } \quad x_{u}(k ; x) \in \lambda \mathcal{F}_{\infty} \quad \forall k \in \mathbb{N}_{0} .
$$

(b) If, in addition, Assumption 3 holds, the origin is contained in the interior of the viability kernel, i.e., $0 \in \operatorname{int} \mathcal{F}_{\infty}$.

Proof. Fix any $\lambda \in(0,1]$. If $\lambda=0$ the result is obvious being 0 an equilibrium. Given any $x \in \lambda \mathcal{F}_{\infty}$ we have that $x / \lambda \in \mathcal{F}_{\infty}$ and thus there is $u_{\lambda} \in \mathcal{U}^{\infty}(x / \lambda)$ such that

$$
\left(x_{u_{\lambda}}(k ; x / \lambda), u_{\lambda}(k)\right) \in \mathcal{E} \quad \text { and } \quad x_{u_{\lambda}}(k ; x / \lambda) \in \mathcal{F}_{\infty} \quad \forall k \in \mathbb{N}_{0}
$$

Define the control sequence $u:=\lambda u_{\lambda}$, we claim that $u \in \mathcal{U}^{\infty}(x)$. By linearity $\lambda x_{u_{\lambda}}(k ; x / \lambda)=x_{u}(k ; x)$ holds and part (a) follows upon multiplication by $\lambda$. Part (b). Since the pair $(A, B)$ is stabilizable, a feedback law $F \in \mathbb{R}^{m \times n}$ exists such that $\varrho(A+B F)<1$ holds, i.e. all eigenvalues of the closed loop 
given by $A+B F$ are contained in the interior of the unit circle, cf. Hinrichsen and Pritchard (2005). As a consequence, constants $C \geq 1$ and $\sigma \in(0,1)$ exist such that, for each state $x_{0} \in \mathbb{R}^{n}$, the closed loop solution $\left(x_{F}\left(k ; x_{0}\right)\right)_{k \in \mathbb{N}_{0}}$ generated by $x_{F}\left(k+1 ; x_{0}\right)=(A+B F) x_{F}\left(k ; x_{0}\right), x_{F}\left(0 ; x_{0}\right)=x_{0}$, satisfies

$$
\left|x_{F}\left(k ; x_{0}\right)\right| \leq\left\|(A+B F)^{k}\right\|\left|x_{0}\right| \leq C \sigma^{k}\left|x_{0}\right| \quad \forall k \in \mathbb{N}_{0}
$$

This shows in particular that $\left|\left(x_{F}\left(k ; x_{0}\right), F x_{F}\left(k ; x_{0}\right)\right)\right| \leq C \sigma^{k}(\|F\|+1)\left|x_{0}\right|$ holds. Recall that $(0,0) \in \operatorname{int} \mathcal{E}$ by hypothesis. Therefore existence of an $\varepsilon$ ball $\varepsilon \mathbb{B} \subseteq \mathcal{E}$ is ensured. Hence, $\left(x_{F}\left(k ; x_{0}\right), F x_{F}\left(k ; x_{0}\right)\right), k \in \mathbb{N}_{0}$, is admissible, which implies $x_{0} \in \mathcal{F}_{\infty}$ for arbitrary $x_{0} \in \delta \mathbb{B}$ with $C(\|F\|+1) \delta \leq \varepsilon$. This completes the proof of the proposition.

According to Propositions 8 and 9, when Assumptions 2 and 3 are in force, the viability kernel $\mathcal{F}_{\infty}$ is a compact and convex set containing the origin in its interior and, for any $\lambda \in[0,1]$, the shrunk set $\lambda \mathcal{F}_{\infty}$ is controlled forward invariant, i.e. for any $x \in \lambda \mathcal{F}_{\infty}$ there exists a feasible state trajectory remaining in $\lambda \mathcal{F}_{\infty}$ for any time. In addition, by the fact that $\mathcal{F}_{\infty}$ is the maximal forward invariant set, we have information about the behaviour of feasible trajectories on $\partial \mathcal{F}_{\infty}$.

Proposition 10. Consider the linear system (L). If $x \in \partial \mathcal{F}_{\infty}$, every feasible trajectory will remain on the boundary unless it touches $\partial X$.

Proof. The result derives from the fact that $\mathcal{F}_{\infty}$ is the maximal forward invariant set. If there were a control $u \in U(x)$ for $x \in \partial \mathcal{F}_{\infty} \backslash \partial X$ such that $f(x, u) \in \operatorname{int} \mathcal{F}_{\infty}$, then by continuity this would be true on a neighbourhood of $x$ making $\mathcal{F}_{\infty}$ larger. For details we refer to Quincampoix (1992).

Remark 11. We remark that Proposition 10 remains true even if a nonlinear system is considered, provided the dynamics $f(\cdot)$ are continuous.

\subsection{Linear Quadratic $M P C$}

The following Proposition provides a uniform bound for $V_{\infty}(\cdot)$ on the interior of the viability kernel. This is a key ingredient in order to characterize the operating range of the MPC feedback law $\mu_{N}$. In particular, the set $\mathcal{O}$ constructed in Subsection 3.2, if nonempty, can contain only points of the boundary of the viability kernel. 
Proposition 12. Let Assumptions 2 and 3 be satisfied and let quadratic running costs $\ell: \mathbb{R}^{n} \times \mathbb{R}^{m} \rightarrow \mathbb{R}_{\geq 0}$ be given by

$$
\ell(x, u):=\left(\begin{array}{ll}
x^{T} & u^{T}
\end{array}\right)\left(\begin{array}{cc}
Q & N \\
N^{T} & R
\end{array}\right)\left(\begin{array}{l}
x \\
u
\end{array}\right)
$$

with symmetric matrices $Q \in \mathbb{R}^{n \times n}$ and $R \in \mathbb{R}^{m \times m}$. Then, for each $\lambda \in[0,1)$ the optimal value function is uniformly bounded from above on $\lambda \mathcal{F}_{\infty}$, i.e., a constant $M=M(\lambda) \in \mathbb{R}_{\geq 0}$ exists such that $V_{\infty}(x) \leq M$ holds for all $x \in \lambda \mathcal{F}_{\infty}$.

Proof. We borrow techniques from (Gondhalekar et al., 2009, Lemma 12). Fix any $\lambda \in(0,1)$ and choose $x \in \lambda \mathcal{F}_{\infty}$ (the result is obvious for $\lambda=0$ ). As we have seen in Proposition 9(a) there exists $u_{I} \in \mathcal{U}^{\infty}(x)$ such that

$$
\left(x_{u_{I}}(k ; x), u_{I}(k)\right) \in \lambda \mathcal{E} \quad \text { and } \quad x_{u_{I}}(k ; x) \in \lambda \mathcal{F}_{\infty} \quad \forall k \in \mathbb{N}_{0},
$$

i.e. a feasible state trajectory which remains in the interior of $\mathcal{F}_{\infty}$. In addition, since $(A, B)$ is stabilizable, a feedback law $F \in \mathbb{R}^{m \times n}$ exists such that the corresponding closed loop $x_{F}^{+}=(A+B F) x_{F}$ satisfies Inequality (16), i.e.

$$
\left|x_{F}(k ; x)\right| \leq\left\|(A+B F)^{k}\right\||x| \leq C \sigma^{k}|x| \quad \forall k \in \mathbb{N}_{0}
$$

for some $C \geq 1$ and $\sigma \in(0,1)$ which, in particular, implies $x_{F}(k ; x) \rightarrow 0$ as $k \rightarrow \infty$. However, the pair $\left(x_{F}, F x_{F}\right)$ may not satisfy the constraints. The idea is to take a convex combination of these two trajectories and exploit linearity and convexity of the data to show that such a combination defines a feasible trajectory which converges to 0 . When a sufficiently small neighbourhood of the origin is reached, the constraints can be neglected and the feedback law $F$ is applied. This procedure yields a uniform bound for $V_{\infty}(\cdot)$. Analytic arguments follow.

Using the control sequence $u_{F}$ given by $u_{F}(k):=F x_{F}(k ; x), k \in \mathbb{N}_{0}$, we have

$$
\left|\left(x_{F}(k ; x), u_{F}(k)\right)\right| \leq C \sigma^{k}(\|F\|+1)|x| \leq L \lambda \sigma^{k} d_{\text {min }} \quad \forall k \in \mathbb{N}_{0}
$$

where $L:=C(\|F\|+1) d_{\max } d_{\text {min }}^{-1}$ with $d_{\text {min }}:=\inf _{x \in \partial X}|x|>0$ and $d_{\max }:=$ $\sup _{x \in X}|x|<\infty$. Hence, $\left(x_{F}(k ; x), u_{F}(k)\right) \in L \lambda \mathcal{E}$ holds for all $k \in \mathbb{N}_{0}$. If $\lambda L \leq 1, u_{F} \in \mathcal{U}^{\infty}(x)$ so that $x_{F}(k ; x)$ is feasible for every $k \in \mathbb{N}_{0}$ and a uniform bound for $V_{\infty}(\cdot)$ is given by $\sup _{x \in X} J_{\infty}\left(x, u_{F}\right) \leq \alpha$ for some $\alpha \in \mathbb{R}_{\geq 0}$. 
Otherwise, for $\lambda L>1$, the control sequence $u$ is defined as $u(k):=$ $\mu u_{I}(k)+(1-\mu) u_{F}(k), k \in \mathbb{N}_{0}$, with $\mu:=\frac{\lambda L-1}{\lambda(L-1)} \in(0,1)$. Then, by linearity of the dynamics

$$
x_{u}(k ; x)=\mu x_{u_{I}}(k ; x)+(1-\mu) x_{u_{F}}(k ; x) .
$$

Our choice of $\mu$ implies $\mu \lambda+(1-\mu) L \lambda=1$ and, thus, $\left(x_{u}(k ; x), u(k)\right) \in \mathcal{E}$ for all $k \in \mathbb{N}_{0}$ which is, in turn, equivalent to admissibility of $u$. Now since $x_{F}(k ; x) \rightarrow 0$ as $k \rightarrow \infty$, if $k$ is taken large enough, the pair $\left(x_{u}(k ; x), u(k)\right) \in$ $\varepsilon \lambda \mathcal{E}$ for some $\varepsilon \in(\mu, 1)$. More precisely, $\left(x_{u}(k ; x), u(k)\right) \in \varepsilon \lambda \mathcal{E}$ holds if (recall Estimate (18))

$$
\mu \lambda+(1-\mu) L \lambda \sigma^{k} \leq \varepsilon \lambda .
$$

Call $k^{*}$ the first integer such that this condition is satisfied. If for example $\varepsilon:=\mu+\frac{1-\mu}{L}$, then $k^{*}$ is such that $\sigma^{k^{*}} \leq \frac{1}{L^{2}}$ and so it is the only integer satisfying $\log _{\sigma}\left(\frac{1}{L^{2}}\right) \leq k^{*}<\log _{\sigma}\left(\frac{1}{L^{2}}\right)+1$.

The point $x_{u}\left(k^{*} ; x\right) \in \lambda \varepsilon \mathcal{F}_{\infty}$ and the procedure followed so far can be iterated, say $m$ times, until $x_{u}\left(m k^{*} ; x\right) \in \lambda \varepsilon^{m} \mathcal{F}_{\infty}$ and $\lambda \varepsilon^{m} L \leq 1$. We keep calling $u$ the admissible sequence that transfers the point $x \in \lambda \mathcal{F}_{\infty}$ to the point $x_{u}\left(m k^{*} ; x\right) \in \lambda \varepsilon^{m} \mathcal{F}_{\infty}$. As soon as the condition $\lambda \varepsilon^{m} L \leq 1$ is satisfied, we switch to the feedback $F$ which ensures that the system feasibly converges to the origin with uniformly bounded costs for each state contained in $\lambda \varepsilon^{m} \mathcal{F}_{\infty}$. Since $X$ and the constraint set are bounded, the prior caused costs are also uniformly bounded since the number of steps needed in order to reach this set is bounded by $m k^{*}$. Note that in particular

$$
V_{\infty}(x) \leq J_{m k^{*}}(x, u)+J_{\infty}\left(x_{u}\left(m k^{*} ; x\right), u_{F}\right) .
$$

This, in addition to the convergence to the origin already shown in (Gondhalekar et al., 2009, Lemma 12), provides bounds on the number of steps required in order to reach an arbitrarily small neighborhood of the origin independent of the chosen initial state $x \in \lambda \mathcal{F}_{\infty}$.

Note that, in Proposition 12, the compactness assumption on the constraint set cannot be dispensed with. For the simple system $x^{+}=2 x+u$, $u \in[-1,1], X=\mathbb{R}$ we have that $\mathcal{F}_{\infty}=\mathbb{R}$ but $V_{\infty}(x)<\infty$ only on the open interval $(-1,1)$.

We are now ready to show that for linear quadratic systems, in Theorem 6, Assumption 1 and Condition (5) can be replaced by the easily checkable 
Assumptions 2 and 3. Moreover, the set $V_{\infty}^{-1}[0,+\infty) \backslash \mathcal{O}$, see Theorem 6 , can be replaced by the interior int $\mathcal{F}_{\infty}$ of the viability kernel. ${ }^{4}$

Theorem 13. Let Assumptions 2 and 3 hold. Furthermore, let the dynamics be given by $(\mathrm{L})$ and the running costs by (17) such that the matrix comprised of $Q, R$, and $N$ is positive definite. Let $K \subseteq \operatorname{int} \mathcal{F}_{\infty}$ be a compact set. Then, a prediction horizon $N_{K} \in \mathbb{N}$ exists such that, for each $N \geq N_{K}$, the $M P C$ feedback law $\mu_{N}$ asymptotically stabilizes the closed loop at $x^{\star}=0$ on a recursively feasible set $\mathcal{S} \supseteq K$.

Proof. Since the running costs $\ell$ are quadratic and positive definite there exist constants $\underline{c}, \bar{c}$ such that $\underline{c}|x|^{2} \leq \ell^{\star}(x) \leq \bar{c}|x|^{2}$, i.e. Condition (5) holds. Furthermore, since the origin is contained in the interior of the constraint set $\mathcal{E}$ and the pair $(A, B)$ is supposed to be stabilizable, a neighborhood $\mathcal{N}$ of the origin exists such that an LQR can be applied neglecting the constraints. Then, the solution $P$ of the algebraic Riccati equation fulfills $V_{\infty}\left(x_{0}\right)=$ $x_{0}^{T} P x_{0} \leq c\left|x_{0}\right|^{2} \leq \rho \ell^{\star}\left(x_{0}\right)$ with $\rho:=c \underline{c}^{-1}$ on $\mathcal{N}$ where $c$ is the maximal eigenvalue of $P$, implying Assumption 1.

Moreover, since $K$ is compact and contained in int $\mathcal{F}_{\infty}$, we can conclude that $K \subseteq \operatorname{int} \lambda \mathcal{F}_{\infty}$ for some $\lambda \in(0,1)$. Hence, by Proposition $12, V_{\infty}$ is bounded on a neighborhood of $K$ and consequently $K \subseteq V_{\infty}^{-1}[0,+\infty) \backslash \mathcal{O}$.

Hence, all assumptions of Theorem 6 are satisfied and the assertion follows from this theorem.

Next, under the assumptions of Theorem 13 we are going to investigate the dependence of the horizon $N_{K}$ on the distance of the compact set $K$ from the boundary of the viability kernel $\partial \mathcal{F}_{\infty}$. To this end, denoting the control $u$ in $(19)$ by $u_{x}$, we obtain

$$
\sup _{x \in \lambda \mathcal{F}_{\infty}} V_{\infty}(x) \leq \sup _{x \in \lambda \mathcal{F}_{\infty}} J_{m k^{*}}\left(x, u_{x}\right)+\sup _{x \in X} J_{\infty}\left(x, u_{F}\right) \leq \beta m k^{*}+\alpha,
$$

for constants $\alpha, \beta>0$ only depending on the data of the problem $(m=0$ holds for $\lambda L \leq 1)$. We emphasise that $m$ depends on $\lambda$, indeed $m$ is the smallest integer which satisfies $\varepsilon^{m} \leq \frac{1}{\lambda L}$. We also recall that $k^{*}$ can be chosen to be the unique integer which satisfies $\log _{\sigma}\left(\frac{1}{L^{2}}\right) \leq k^{*}<\log _{\sigma}\left(\frac{1}{L^{2}}\right)+$

\footnotetext{
${ }^{4}$ Observe that $\mathcal{O}$ may still contain points on $\partial \mathcal{F}_{\infty}$ at which the value function is finite but discontinuous, cf. Boccia et al. (2014) for details.
} 
1. Consequently defining $\tilde{\beta}:=\beta k^{*}$ we obtain $\sup _{x \in \lambda \mathcal{F}_{\infty}} V_{\infty}(x) \leq \tilde{\beta} m+\alpha$. Following our choice $\varepsilon=\mu+\frac{1-\mu}{L}$ (where we recall $\mu=\frac{\lambda L-1}{\lambda(L-1)}$ ) we have that $\varepsilon=1-\frac{1-\lambda}{\lambda L}$. This yields an estimate for the growth of $m$. More precisely

$$
m \sim \frac{L \ln L}{1-\lambda} \quad \text { as } \lambda \rightarrow 1 .
$$

This estimates is achieved by using the definition of $m$ as the smallest integer which satisfies $\varepsilon^{m} \leq \frac{1}{\lambda L}$, and performing an asymptotic analysis.

When we are in a sufficiently small neighbourhood of the origin, say $\delta \mathbb{B}$, constraints can be neglected and $V_{\infty}(x)=x^{T} P x$ where $P$ is the solution of the algebraic Riccati equation. This in turn gives a bound for $V_{\infty}(\cdot)$ of the type $V_{\infty}(x) \leq \rho|x|^{2}$ for all $x \in \delta \mathbb{B}$. Away from the origin, when constraints are present this bound is no longer satisfied. We have shown, though, that it is possible to find constants $\tilde{\beta}$ and $\tilde{\alpha}:=\max \{\rho, \alpha / \delta\}$ such that

$$
V_{\infty}(x) \leq \tilde{\beta} m(x)+\tilde{\alpha}|x|^{2} .
$$

Here $m(x):=\inf \left\{m \in \mathbb{N}:\left(1-\frac{1-g(x)}{g(x) L}\right)^{m} \leq \frac{1}{g(x) L}\right\}$, where

$$
g(x):=\inf \left\{\gamma>0: \gamma^{-1} x \in \mathcal{F}_{\infty}\right\} .
$$

By definition, $m(x)=+\infty$ if $x \notin \operatorname{int} \mathcal{F}_{\infty}$ (or equivalently if $g(x) \geq 1$ ), $m(x)=0$ if $g(x) \leq 1 / L$ and it is a finite natural number elsewhere. Moreover by (20) we know that $\lim _{x \rightarrow \partial \mathcal{F}_{\infty}} m(x)=+\infty$. In the next corollary we give a characterization of the asymptotic behavior of $m(x)$ (and hence of $V_{\infty}(x)$ ) in terms of the distance of $x$ from $\partial \mathcal{F}_{\infty}$ when $x$ approaches the boundary of $\mathcal{F}_{\infty}$.

Corollary 14. Let the assumptions of Proposition 12 be satisfied. Then the function $m($.$) , defined in (21), behaves asymptotically like { }^{5} m(x) \sim \frac{\omega L \ln L}{\operatorname{dist}\left(x ; \partial \mathcal{F}_{\infty}\right)}$ as $x \rightarrow \partial \mathcal{F}_{\infty}$, where $\omega \in\left[\inf _{x \in \partial \mathcal{F}_{\infty}}|x|, \sup _{x \in \mathcal{F}_{\infty}}|x|\right]$ and $L$ is from the proof of Proposition 12. Moreover, $m(x)=0$ in a sufficiently small neighbourhood of the origin.

Proof. We only have to verify the assertion $m(x) \sim \frac{\omega L \ln L}{\operatorname{dist}\left(x ; \partial \mathcal{F}_{\infty}\right)}$ for some $\omega \in$ $\left[f^{-}, f^{+}\right]$where $f^{-}:=\inf _{x \in \partial \mathcal{F}_{\infty}}|x|$ and $f^{+}:=\sup _{x \in \mathcal{F}_{\infty}}|x|$. To this end we will

\footnotetext{
${ }^{5}$ Given any set $\Omega \subset \mathbb{R}^{n}$ and $x \in \mathbb{R}^{n}$, dist $(x ; \Omega)$ denotes the Euclidean distance of the point $x$ from the set $\Omega$. Given a second set $K \subset \mathbb{R}^{n}, \operatorname{dist}(K ; \Omega):=\min _{x \in K} \operatorname{dist}(x ; \Omega)$.
} 
use the function $g(\cdot)$, defined in (22), to compare $\lambda$ and $\operatorname{dist}\left(x ; \partial \mathcal{F}_{\infty}\right)$ when $x \in \lambda \partial \mathcal{F}_{\infty}{ }^{6}$

For every $x \in \lambda \partial \mathcal{F}_{\infty}, \lambda \in(0,1)$, we define $\pi(x)$ to be a projection of $x$ onto $\partial \mathcal{F}_{\infty}$. Then $\operatorname{dist}\left(x ; \partial \mathcal{F}_{\infty}\right)=|x-\pi(x)|$. Moreover $g(x)=\lambda$ and $g(\pi(x))=1$. Therefore

$$
1-\lambda=g(\pi(x))-g(x) \leq \frac{1}{f^{-}}|x-\pi(x)|=\frac{1}{f^{-}} \operatorname{dist}\left(x ; \partial \mathcal{F}_{\infty}\right) .
$$

On the other hand

$$
\operatorname{dist}\left(x ; \partial \mathcal{F}_{\infty}\right) \leq\left|\frac{x}{\lambda}-x\right| \leq f^{+} \frac{1-\lambda}{\lambda} .
$$

Now the assertion follows from (20).

Using this estimate we arrive at the following estimate for the optimization horizon needed in order to ensure $K \subseteq \mathcal{S}$.

Corollary 15. Given a compact set $K \subseteq \operatorname{int} \mathcal{F}_{\infty}$ there exists a constant $D$ only depending on the data of the problem such that

$$
\sup _{x \in K} V_{\infty}(x) \leq \frac{D}{\operatorname{dist}\left(K ; \partial F_{\infty}\right)} .
$$

Moreover, whenever $N \geq N_{K}$, where $N_{K}$ is the smallest integer satisfying

$$
N_{K}>2+\frac{\ln (\beta-1)}{\ln \beta-\ln (\beta-1)}
$$

for $\beta=\max \left\{D \cdot\left(M \cdot \operatorname{dist}\left(K ; \partial F_{\infty}\right)\right)^{-1}, \gamma\right\}$, then the MPC closed loop is asymptotically stable with recursively feasible basin of attraction $\mathcal{S} \supseteq K$. Asymptotically, $N_{K}$ behaves like

$$
\frac{D}{M \cdot \operatorname{dist}\left(K ; \partial F_{\infty}\right)} \ln \left(\frac{D}{\operatorname{dist}\left(K ; \partial F_{\infty}\right)}\right) .
$$

Proof. The bound in (23) follows directly from Corollary 14 choosing a constant $D$ sufficiently large. Theorem 4 and Remark 5 then yield the inequalities for $N_{K}$.

\footnotetext{
${ }^{6}$ This function is Lipschitz with Lipschitz constant $1 / f^{-}$. (The function $g(\cdot)$ is commonly called gauge or Minkowski functional of $\mathcal{F}_{\infty}$, see Brezis (2011), Lemma 1.2.
} 
The following example illustrates that the required prediction horizon grows rapidly for initial values approaching the boundary of the viability kernel.

Example 16. We consider the controllable and, thus, in particular stabilizable linear system given by

$$
x^{+}=\left(\begin{array}{ll}
1 & 1 \\
0 & 2
\end{array}\right)\left(\begin{array}{l}
x_{1} \\
x_{2}
\end{array}\right)+\left(\begin{array}{ll}
1 & 0 \\
0 & 1
\end{array}\right)\left(\begin{array}{l}
u_{1} \\
u_{2}
\end{array}\right)
$$

with constraints $X:=[-100,100] \times[-1,1]$ and

$$
U:=\left\{u \in \mathbb{R}^{2}:\left(\begin{array}{rrrr}
1 & -1 & 1 & -1 \\
1 & 1 & -1 & -1
\end{array}\right)^{T}\left(\begin{array}{l}
u_{1} \\
u_{2}
\end{array}\right) \leq\left(\begin{array}{llll}
1 & 1 & 1 & 1
\end{array}\right)^{T}\right\} .
$$

The quadratic stage costs are given by

$$
\ell(x, u)=\left(\begin{array}{l}
x_{1} \\
x_{2}
\end{array}\right)^{T}\left(\begin{array}{cc}
100 & 0 \\
0 & 1
\end{array}\right)\left(\begin{array}{l}
x_{1} \\
x_{2}
\end{array}\right)+\left(\begin{array}{l}
u_{1} \\
u_{2}
\end{array}\right)^{T}\left(\begin{array}{cc}
1 & 0 \\
0 & 100
\end{array}\right)\left(\begin{array}{l}
u_{1} \\
u_{2}
\end{array}\right) .
$$

Then, the minimal stabilizing horizon $\widehat{N}:=\min \left\{N \in \mathbb{N}: x_{\mu_{N}}\left(k ; x_{0}\right) \rightarrow\right.$ 0 for $k \rightarrow \infty\}$ w.r.t. the origin (controlled equilibrium for $\left.u^{\star}=\left(\begin{array}{ll}0 & 0\end{array}\right)^{T}\right)$ in dependence of given initial values are shown in the following table. ${ }^{7}$

\begin{tabular}{|r|r|r|r|r|r|r|}
\hline$x_{1}$ & 0.5 & 0.5 & 0.5 & 0.5 & 0.5 & 0.5 \\
\hline$x_{2}$ & 0.9 & 0.99 & 0.999 & 0.9999 & 0.99999 & 0.999999 \\
\hline$\widehat{N}$ & 6 & 6 & 7 & 10 & 13 & 16 \\
\hline
\end{tabular}

\subsection{Boundedness of $V_{\infty}$ on the Viability Kernel $\mathcal{F}_{\infty}$}

In the preceeding Subsection 4.2 we considered the stabilization task for arbitrary compact sets contained in the interior of the viability kernel $\mathcal{F}_{\infty}$. However, it follows from Theorem 4 that for each sufficiently large $N$ MPC will yield asymptotic stability with the basin of attraction $\mathcal{S}$ containing the whole viability kernel $\mathcal{F}_{\infty}$ if $\sup V_{\infty}\left(\mathcal{F}_{\infty}\right)$ is finite. In this final section we show that this property implies stationarity of the feasible sets $\mathcal{F}_{N}$.

\footnotetext{
${ }^{7}$ Note that the point $(0.5,1)^{T}$ is not contained in the viability kernel $\mathcal{F}_{\infty}$. $\widehat{N}$ was computed with the Matlab-routine mpc_ExampleBGW.m which is available for download at http://num.math.uni-bayreuth.de/de/publications/2014/boccia_et_al_feasibility_2014.
} 
We say that the feasible sets $\mathcal{F}_{N}$ become stationary, if there exists $N_{0} \in \mathbb{N}$ with $\mathcal{F}_{N}=\mathcal{F}_{N_{0}}$ for all $N \geq N_{0}$. In (Kerrigan, 2000, Theorem 5.3) (see also (Grüne, 2012, Section 5.1)), it was shown that stationarity of the feasible sets is sufficient for recursive feasibility of $\mathcal{F}_{\infty}$ for all optimization horizons $N \geq N_{0}+1$. In the following theorem we show that it is also necessary for $V_{\infty}$ being bounded on the viability kernel $\mathcal{F}_{\infty}$.

Theorem 17. Consider the linear system $(L)$ with positive definite quadratic running costs $\ell$ and let Assumptions 2 and 3 be satisfied. Then, if $V_{\infty}(x) \leq$ $c$ holds for some $c \in \mathbb{R}_{>0}$ and all $x \in \mathcal{F}_{\infty}$, the feasible sets $\mathcal{F}_{N}$ become stationary for some $N_{0} \in \mathbb{N}$.

Proof. By definition $\mathcal{F}_{N} \supseteq \mathcal{F}_{\infty}$. Moreover we know that, for every $N \in \mathbb{N}$, $\mathcal{F}_{N}$ is convex (Proposition 8) and that $V_{N}$ is a convex function (convex costs, convex constraints, and linear dynamics). We prove the result by showing the existence of $N_{0}$ with $\mathcal{F}_{N_{0}}=\mathcal{F}_{\infty}$, which implies stationarity. We proceed by contradiction, i.e., we assume that $\mathcal{F}_{N} \supsetneq \mathcal{F}_{\infty}$ holds for every $N \in \mathbb{N}$.

Due to Assumptions 2 and 3 and the linearity of the system dynamics $\mathcal{F}_{\infty}$ contains a (small) ball $\mathcal{B}_{r}:=\left\{x \in \mathbb{R}^{n}:\|x\|<r\right\}$ with radius $r>0$ around the origin. Hence, any trajectory originating at $x_{0}$ cannot reach $\mathcal{F}_{\infty}$ and in particular remains outside $\mathcal{B}_{r}$. Then, if $N \in \mathbb{N}$ is chosen sufficiently large, we have that $V_{N}\left(x_{0}\right)>c+2$ for every $x_{0} \in \mathcal{F}_{N} \backslash \mathcal{F}_{\infty}$ since the running costs $\ell(\cdot)$ imply costs of at least $\inf _{x \in X:\|x\| \geq r} \ell^{\star}(x)=: \varrho>0$ along the respective optimal trajectory and, thus, $V_{N}\left(x_{0}\right) \geq N \varrho$ holds.

Now fix a natural number $N \in \mathbb{N}$ with $N \varrho>c+2$ and observe that by convexity of the set $\mathcal{F}_{N}$ we may choose $x \in \mathcal{F}_{N} \backslash \mathcal{F}_{\infty}$ and $y \in \partial \mathcal{F}_{\infty}$ such that $\lambda y+(1-\lambda) x \in \mathcal{F}_{N} \backslash \mathcal{F}_{\infty}$ for all $\lambda \in(0,1)$. This implies the inequalities $V_{N}(\lambda y+(1-\lambda) x)>c+2$ for all $\lambda \in(0,1)$ and $V_{N}(y) \leq V_{\infty}(y) \leq c$. Then for all $\lambda \in(0,1)$, convexity of $V_{N}$ yields

$$
c+2<V_{N}(\lambda y+(1-\lambda) x) \leq \lambda V_{N}(y)+(1-\lambda) V_{N}(x) \leq \lambda c+(1-\lambda) V_{N}(x) .
$$

For $\lambda$ sufficiently close to 1 we obtain the desired contradiction because $V_{N}(x)$ is finite.

The converse is not true in general as shown in the following Example 18.

Example 18. Consider the discrete time system in $\mathbb{R}$ given by

$$
x^{+}=2 x+u \quad \text { with constraint set } \mathcal{E}:=[-1,1] \times[-1,1] .
$$


Since every $x \in X=[-1,1]$ is a controlled equilibrium $(u=-x) \mathcal{F}_{\infty}=X$ and, thus, $\mathcal{F}_{N}=\mathcal{F}_{\infty}$ actually holds for every $N \in \mathbb{N}$. Yet, for any positive definite quadratic cost $V_{\infty}$ fails to be bounded on $\partial \mathcal{F}_{\infty}$ and grows unboundedly for $x \rightarrow \partial \mathcal{F}_{\infty}$, as the following computation shows.

If $x_{0}=1$ the only admissible control sequence $u$ is $u \equiv-1$ for every time instant. Indeed $x_{u}(k ; 1)=1$ for every $k \in \mathbb{N}$. Therefore as soon as we define a cost say $\ell(x, u)=x^{2}$ we have that $V_{\infty}(1)=+\infty$. The point $x_{0}=-1$ has a similar behaviour. Every other initial point $x_{0} \in(-1,1)=X \backslash\{1,-1\}$, different from 1 and -1 , can be controlled to zero in finite time by

$$
u_{x_{0}}(k)=-\operatorname{sign}\left(x_{u_{x_{0}}}\left(k ; x_{0}\right)\right) \min \left\{2\left|x_{u_{x_{0}}}\left(k ; x_{0}\right)\right|, 1\right\} .
$$

However, the closer $x_{0}$ to 1 or -1 , the longer it will take before an interval of the form $[-\delta, \delta]$ for $\delta \in(0,1)$ can be reached. Hence, as $x_{0} \rightarrow 1$ or $x_{0} \rightarrow-1$, the value function $V_{\infty}\left(x_{0}\right)$ tends to $+\infty$.

\section{Conclusions}

We investigated recursive feasibility and asymptotic stability for nonlinear MPC schemes with state and control constraints without imposing stabilizing terminal constraints or costs. Assuming a local controllability condition around the equilibrium to be stabilized, we have shown that the system can be asymptotically stabilized and that any level set $V^{-1}[0, C]$ of the infinite horizon optimal value function $V_{\infty}$ is contained in the domain of attraction for sufficiently large optimization horizon $N$. Moreover, we established a quantitative relation between the level $C$ and the proposed estimate of the horizon $N$.

For linear systems, this relation was made more precise by estimating the growth of $V_{\infty}$ at the boundary of the viability kernel. Particularly, we have shown that any compact subset of the interior of the viability kernel $\mathcal{F}_{\infty}$ is contained in the basin of attraction $\mathcal{S}$ for sufficiently large $N$. Our analysis moreover shows that the whole viability kernel $\mathcal{F}_{\infty}$ is contained in $\mathcal{S}$ if $V_{\infty}$ is bounded on $\mathcal{F}_{\infty}$. This property, in turn, implies stationarity of the feasible $\operatorname{sets} \mathcal{F}_{N}$.

\section{Acknowledgment}

This work was supported by the European Union under the 7th Framework Programme FP7-PEOPLE-2010-ITN Grant agreement number 264735SADCO (A. Boccia and L. Grüne) and by the DFG Grant GR1569/12-2 (K. 
Worthmann). The research for this paper was carried out while the first author visited the University of Bayreuth during his SADCO secondment.

\section{References}

Aubin, J.P., Frankowska, H., 1990. Set-valued analysis. volume 2 of Systems 8 Control: Foundations 83 Applications. Birkhäuser Boston Inc., Boston, MA.

Blanchini, F., Miani, S., 2008. Set-Theoretic Methods in Control. Birkhäuser.

Boccia, A., Grüne, L., Worthmann, K., 2014. Stability and feasibility of state-constrained linear MPC without stabilizing terminal constraints, in: Proceedings of the 21st International Symposium on Mathematical Theory of Networks and Systems (MTNS2014), pp. 453-460.

Brezis, H., 2011. Functional analysis, Sobolev spaces and partial differential equations. Universitext, Springer, New York.

Gondhalekar, R., Imura, J., Kashima, K., 2009. Controlled invariant feasibility - A general approach to enforcing strong feasibility in MPC applied to move-blocking. Automatica 45, 2869-2875.

Grimm, G., Messina, M., Tuna, S., Teel, A., 2005. Model predictive control: for want of a local control Lyapunov function, all is not lost. IEEE Trans. Automat. Control 50, 546-558.

Grüne, L., 2009. Analysis and design of unconstrained nonlinear MPC schemes for finite and infinite dimensional systems. SIAM J. Control Optim. 48, 1206-1228.

Grüne, L., 2012. NMPC without terminal constraints, in: Proceedings of the IFAC Conference on Nonlinear Model Predictive Control 2012 (NMPC'12), pp. 1-13.

Grüne, L., Pannek, J., 2011. Nonlinear Model Predictive Control: Theory and Algorithms. Communications and Control Engineering, Springer London Dordrecht Heidelberg New York.

Grüne, L., Pannek, J., Seehafer, M., Worthmann, K., 2010. Analysis of unconstrained nonlinear MPC schemes with varying control horizon. SIAM J. Control Optim. 48 (8), 4938-4962. 
Hinrichsen, D., Pritchard, A., 2005. Mathematical Systems Theory I. Number 48 in Texts in Applied Mathematics, Springer, Berlin Heidelberg.

Jadbabaie, A., Hauser, J., 2005. On the stability of receding horizon control with a general terminal cost. IEEE Transactions on Automatic Control $50,674-678$.

Kerrigan, E.C., 2000. Robust constraint satisfaction: Invariant sets and predictive control. PhD Thesis, University of Cambridge.

Mayne, D., 2013. An apologia for stabilising terminal conditions in model predictive control. International Journal of Control 86, 2090-2095.

Primbs, J., Nevistić, V., 2000. Feasibility and stability of constrained finite receding horizon control. Automatica 36, 965-971.

Quincampoix, M., 1992. Differential inclusions and target problems. SIAM J. Control Optim. 30 (2), 324-335.

Raff, T., Huber, S., Nagy, Z.K., Allgöwer, F., 2006. Nonlinear model predictive control of a four tank system: An experimental stability study, in: Proceedings of the IEEE Conference on Control Applications, Munich, Germany. pp. 237-242.

Tuna, S.E., Messina, M.J., Teel, A.R., 2006. Shorter horizons for model predictive control, in: Proceedings of the American Control Conference, Minneapolis, Minnesota, USA. pp. 863-868.

Worthmann, K., 2011. Stability Analysis of unconstrained Receding Horizon Control. Ph.D. thesis. University of Bayreuth. 